\title{
Study of seismic attributes recognition method of high quality igneous rock reservoir
}

\author{
Wang Yitong ${ }^{1,2}$, Li Jingsheng ${ }^{1,2}$, Sam Zandong Sun ${ }^{3}$, Qiao Wei ${ }^{1,2}$, Li Yanjie ${ }^{1,2}$, Ma Yongyi ${ }^{1,2}$, Shen Yan ${ }^{1,2}$ and \\ Wang Shuheng ${ }^{1,2}$ \\ ${ }^{1}$ Exploration and Development Research Institute of Daqing Oilfield Co., Ltd., 163712 Daqing Heilongjiang, China \\ ${ }^{2}$ Key Laboratory of tight oil and shale oil accumulation of Heilongjiang province, 163712 Daqing Heilongjiang, China \\ ${ }^{3}$ BGP, 052751 Zhuozhou Hebei, China
}

\begin{abstract}
Aimed target area is deeply buried, complex lithology, dual media, reservoir development degree is controlled by a variety of factors, meanwhile, lateral thickness and lithofacies change rapidly, and strata formation is poor. Therefore, igneous rock reservoir has difficulty in predicting, since seismic is complicated to track trace, reservoir attribute analysis is hard to determine the time window, and inversion modeling requires sophisticated. By analyzing, the basalt in the target research area accounts for the principal component of the igneous rock, however, the igneous rocks with relatively developed reservoirs are mostly distributed in the trachyte breccia which has good productivity. The results of petrophysical study indicate that frequency-dependent AVO inversion method is an important means to identify fluid and reservoir prediction, notwithstanding it is difficult to distinguish high-quality reservoirs barely by P-wave impedance. Consequently, AVOF inversion method is appropriately proposed to identify igneous rock reservoir. Foremost, eliminating the effects of algorithm, frequency, spectrum balancing and other factors, then put the improved three-term Aki\&Richards frequency-dependent AVO inversion method applying to distinguish igneous reservoir fluid and lithology, for the purpose of carrying out the identification of high-quality reservoirs.
\end{abstract}

\section{Introduction}

Mason and Mcskinin first pointed out the relationship between wavelength attenuation and frequency by measuring the acoustic attenuation of some metals and glasses and the relationship between frequency and particle size [1]. Toulis and Shumway measured the acoustic velocity and attenuation of sedimentary rocks by resonance method, which was an earlier experimental measurement to study the relationship between frequency, velocity and attenuation when waves propagate in sedimentary rocks [2]. Chapman proposed that the seismic wave reflection coefficient changes with frequency and has a great relationship with the type of AVO [3]. Wilson established a practical method for calculating the dispersion properties [4]. Wu Xiaoyang calculated seismic dispersion values from actual data and achieved good results [5]. An improved algorithm by employing Aki \&Richards approximation to obtain more accurate inverted P-wave dispersion was presented and analyzed multiple technical steps affecting dispersion, such as amplitude-preserved processing, high-resolution time-frequency analysis technology, frequency components and reference frequency [6,7]. Both physical model test and real seismic example indicating that the magnitude of P-wave dispersion among three typical fluids (i.e. gas, oil and water) are obviously different, concretely, strong, moderate, weak for gas, oil and water respectively [8].

Conventional AVO inversion, ignoring the dispersion phenomenon of fluid, cannot be accurately distinguished from fluid type and lithology. In spite of post-stack methods such as spectral decomposition can be used to point hydrocarbon in seismic data, these type of methods still have many limitations. For instance, incorrect superposition may produce false "low frequency anomalies" and suppress high frequency information; or introducing large offset information that is stretched by dynamic correction during the superposition process, thereby weakening useful information and making the superposition profile, which will hide low frequency information. Additionally, due to the presence of seismic resolution processed by post-stack methods occasionally cannot meet the requirements of reservoir prediction. For this reason, exploring more effective fluid identification and detection methods is indispensable and significant. Considering that the fluid in the reservoir causes the seismic wave dispersion effect, we propose a pre-stack frequency-dependent AVO inversion method based on the more accurate three-term formula Aki \& Richards algorithm with variational $\mathrm{Vs} / \mathrm{Vp}$ obtained from pre-stack AVO inversion to substitute traditional Smith \& Gidlow two-term inversion approximate formula with constant $\mathrm{Vs} / \mathrm{Vp}(0.5)$. The modified method expands the

\footnotetext{
*Corresponding author: wangyitong_sj@foxmail.com
} 
approximate formula of the Zoeppritz equation based on the pre-stack AVO inversion, introduces the frequency term, and solves the dispersion value. The experiments and field data massively demonstrate that the improved frequency-dependent AVO inversion method provides more reliable P-wave dispersion gradient, can more effectively indicate fluid types and cumulative production and suppress non-reservoir energy, paramountly, it can achieve the role of identifying high-quality reservoirs.

\section{Workflow}

The proposed workflow is based on the intergration of different angle stack seismic data and is composed of four processing stages under the supervision of Chapman rock physic theory.

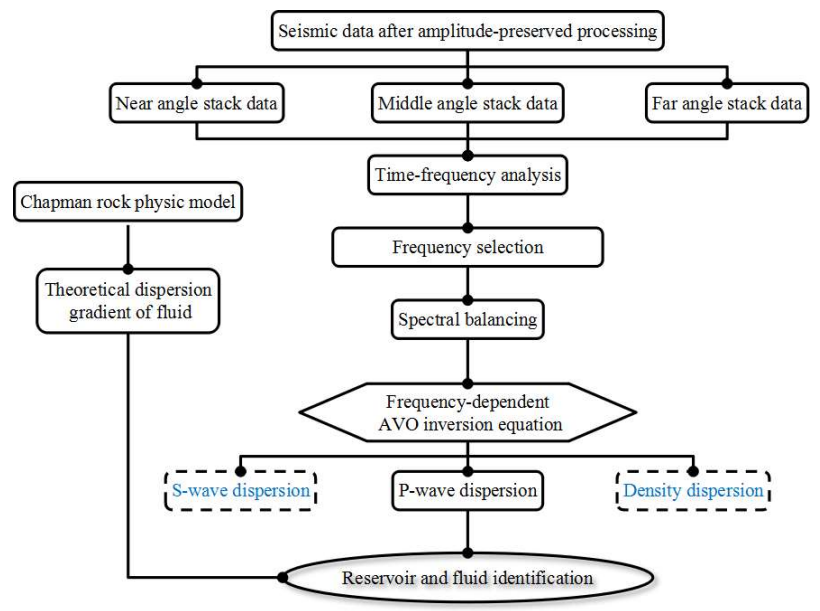

Fig. 1. Workflow of frequency-dependent AVO inversion.

\section{Affecting factors}

\subsection{Factor of model}

Three types of frequency-dependent AVO inversion algorithms are selected to further explore the influence of frequency dispersion results. Well 19 and Well 16 are oil wells with medium yield, and Well 3 and Well 1 are water wells. Compared figure 2(a) with figure 2(b) and figure 2(c), it is obvious that $\mathrm{P}$-wave dispersion gradient values at the two oil wells is higher, and the dispersion response is much stronger, which cannot meet the actual production, as well as poorer suppression of non-reservoir, especially near the water well. The non-reservoir in figure 2(c) has a better suppression effect and fluid dispersion indicator can describe the wells' production more elaborately by comparison with figuir 2(b).

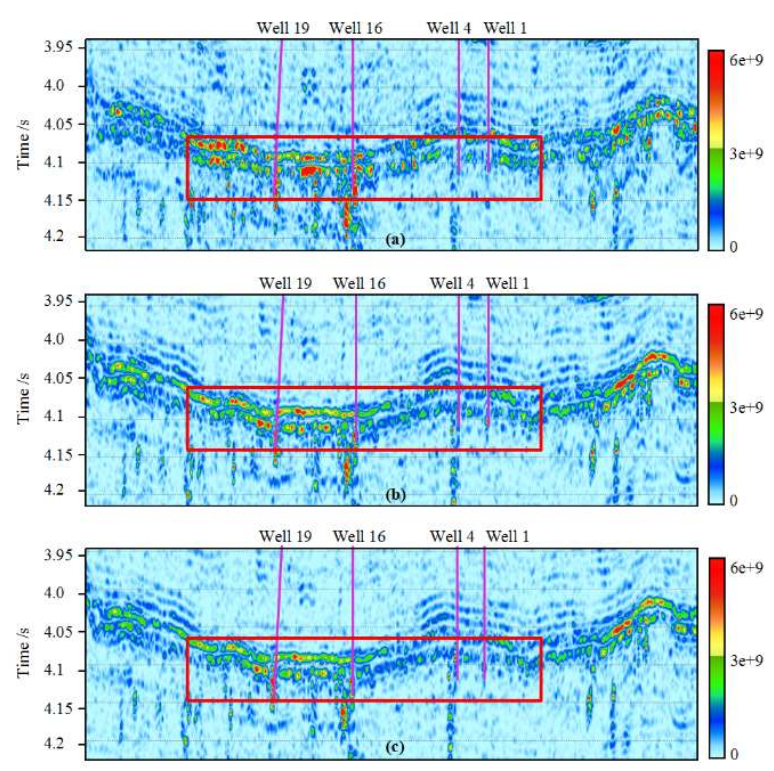

frequency-dependent AVO inversion algorithms

((a) Smith \& Gidlow approximation with constant-Vs/Vp;

(b) Aki \& Richards approximation with constant-Vs/Vp;

(c) Aki \& Richards approximation with variant-Vs/Vp)

Fig. 2 P-wave dispersion gradient sections comparison by different

Figure 3 shows the RMS P-wave dispersion values of three frequency-dependent AVO inversion algorithms. Wells of circles represent P-wave dispersion gradient is inconsistent with oil yield. Table 1 shows the daily oil production of 30 wells, which comprised 25 oil wells including 17 high-yield oil wells, besides 5 water wells. Well 1 and well 24 are water wells, well 15, well 16 and well 20 are low-yield oil wells, as well as well 28 is highyield oil wells. During the detection of fluid types, through different types of inversion algorithms, an image of P-wave dispersion is obtained from various approximate Zoeppritz formulas. It is illustrated that whether water or oil wells display distinct discrepancy between dispersion response and fluid type in figure3(a) comparison of the three obtained results. Despite P-wave dispersion gradient in figure 3(c) does not efficaciously detect Well 20 and Well 24 influenced by faults, in contrast with figure $3(\mathrm{~b})$, the fluid characterization conformance is more accurate about low-yield well 15 and well 16. 

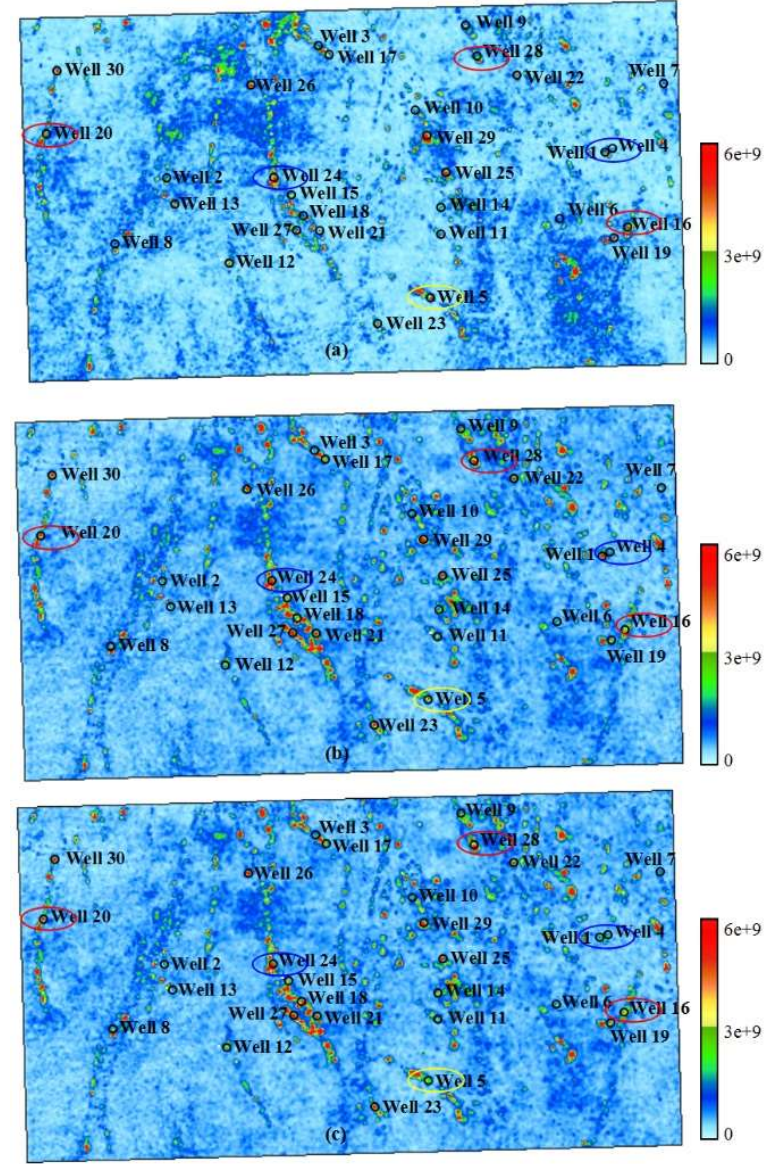

frequency-dependent AVO inversion algorithms

((a) Smith \& Gidlow 2-iterm approximation with constant$\mathrm{Vs} / \mathrm{Vp}$;

(b) Aki \& Richards 3-iterm approximation with constant$\mathrm{Vs} / \mathrm{Vp}$;

(c) Aki \& Richards3-iterm approximation with variant-Vs/Vp).

Fig. 3. P-wave dispersion gradient attributes comparison by different

Table 1. Well production statistics.

\begin{tabular}{|c|c|c|c|}
\hline Well name & $\begin{array}{c}\text { Production } \\
(\mathrm{t} / \text { day })\end{array}$ & Well name & $\begin{array}{c}\text { Production } \\
(\mathrm{t} / \text { day })\end{array}$ \\
\hline Well 1 & 0.91 & Well 16 & 10.58 \\
\hline Well 2 & 1.17 & Well 17 & 10.54 \\
\hline Well 3 & 0.72 & Well 18 & 10.85 \\
\hline Well 4 & 0.51 & Well 19 & 11.09 \\
\hline Well 5 & 1.13 & Well 20 & 7.13 \\
\hline Well 6 & 1.75 & Well 21 & 6.38 \\
\hline Well 7 & 1.66 & Well 22 & 5.40 \\
\hline Well 8 & 4.91 & Well 23 & 17.03 \\
\hline Well 9 & 5.09 & Well 24 & 19.42 \\
\hline Well 10 & 5.99 & Well 25 & 28.74 \\
\hline Well 11 & 7.15 & Well 26 & 22.94 \\
\hline Well 12 & 8.52 & Well 27 & 24.27 \\
\hline Well 13 & 8.98 & Well 28 & 28.43 \\
\hline Well 14 & 9.79 & Well 29 & 28.31 \\
\hline Well 15 & 10.03 & Well 30 & 34.14 \\
\hline
\end{tabular}

*production of $1 \mathrm{~mm}$ nozzle

Analyzing the coincidence rate statistically between Pwave dispersion gradient and the well production, the agreement applying Smith \& Gidlow approximate formula with constant value $\mathrm{Vs} / \mathrm{Vp}$ is $66.7 \%$, the accordance introducing Aki \& Richards approximate formula with constant value $\mathrm{Vs} / \mathrm{Vp}$ is $76.7 \%$, moreover, the rate of Aki \& Richards approximate formula with variable value $\mathrm{Vs} / \mathrm{Vp}$ is $86.7 \%$, which prominently demonstrate that frequency-dependent AVO inversion model will affect the $\mathrm{P}$-wave dispersion gradient results. Generally speaking, the P-wave dispersion gradient calculated based on the Aki \& Richards three-term approximation formula with varying $\mathrm{Vs} / \mathrm{Vp}$ can reflect the fluid type more veritably and effectively.

\subsection{Spectral balancing}

For a certain seismic data volume $\mathrm{s}(\mathrm{t}, \mathrm{n})$, there are $\mathrm{t}$ sampling points on each seismic trace, and timefrequency transform method is performed to obtain a series of single-frequency data volume $\mathrm{S}(\mathrm{t}, \mathrm{n}, \mathrm{f})$

$$
s(t, n) \leftrightarrow S(t, n, f)
$$

For the purpose of eliminating the energy difference of the seismic amplitude spectrum corresponding to the different frequency, it is fundamentally to allow energy "compensation" for each single-frequency amplitude spectrum. This compensation process is called spectrum equalization.

$$
S_{b}(t, n, f)=S(t, n, f) \omega(n, f)
$$

In the above formula, $\omega(n, f)$ is weighting factor responding to relative single-frequency amplitude spectrum data volumes:

$$
\omega(n, f)=\frac{\max \left(A_{r e f}(n)\right)}{\max \left(A_{f}(n)\right)}
$$

At the position of channel $\mathrm{n}, \max \left(A_{\text {ref }}(n)\right)$ refers to the maximum amplitude value of reference frequency $f_{0}$, and $\max \left(A_{f}(n)\right)$ refers to the maximum amplitude value of the single frequency $f$.

The weight function is acquired through an appropriate elastic model. According to Gassmann's theory, the reflection coefficient obtained from the elastic interface is independent of frequency in the seismic frequency band. For dispersion model, there is no such assumption. (The weight function under the elastic model is used to spectraLly equalize the amplitude spectrum of the dispersion model.) That is, in field case, the elastic interface can be regard as a reference to equalize the amplitude spectrum of the dispersive medium. In practical applications, selecting a continuous strong lithological interface (theoretically assumed to be an elastic interface) as the reference interface to perform spectral equalization in the frequency-dependent AVO inversion, and the sampling points of each channel of the frequency division data volume by time-frequency transform method are weighed to obviate the energy discrepancy of the frequency-divided data itself, thereby highlighting the true difference of geological frequency on the seismic signal.

Figure 4 shows the seismic profiles and the P-wave dispersion gradient profiles without and with spectral equalization of the connected wells. The blue circle 
represents water well and the remaining three are oil wells. The P-wave dispersion gradient profile processed by spectrum equalization in figure $4(\mathrm{c})$ is more consistent with the well type. The obtained results show more details that seismic amplitude and $\mathrm{P}$-wave dispersion gradient without spectral balancing process neither can distinguish oil wells from water wells, and has poorer suppression effect on non-reservoir, which is not conducive to subsequent fluid prediction. Moreover, this is consistent with theoretical analysis that it is prerequisite to perform spectrum equalization in the frequency-dependent AVO inversion process.
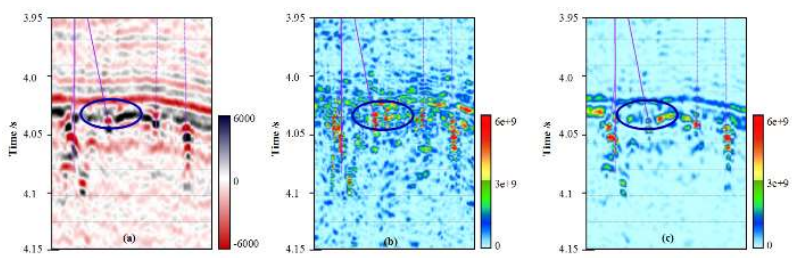

((a) seismic amplitude ; (b) P-wave dispersion gradient with spectral balancing; (c) P-wave dispersion gradient without spectral balancing).

Fig. 4. Comparison of sections

The results show that the diversity of P-wave dispersion gradient indication area in the two plans is significantly (Fig. 5). On the basis of the well production information, the high $\mathrm{P}$-wave dispersion in figure 5(b) has better consistency of the oil and gas production such as Well 18, Well 21, Well 28 and Well 27, additionally, it has better recognition effect for water wells such as well 2 . Not only the suppression effect of non-reservoir is better, but the trend of the high frequency dispersion is similar to that of the fault. It demonstrates that the spectrum equalization processing is validated to eliminate the interference of the frequency-divided data itself to a certain extent, thus highlighting the real seismic frequency response characteristics under geological conditions. Practical applications show that spectrum equalization processing is performed has a great influence on the frequencydependent AVO inversion result. The P-wave dispersion gradient value executed by spectral equalization process can more accurately identify the fluid type.
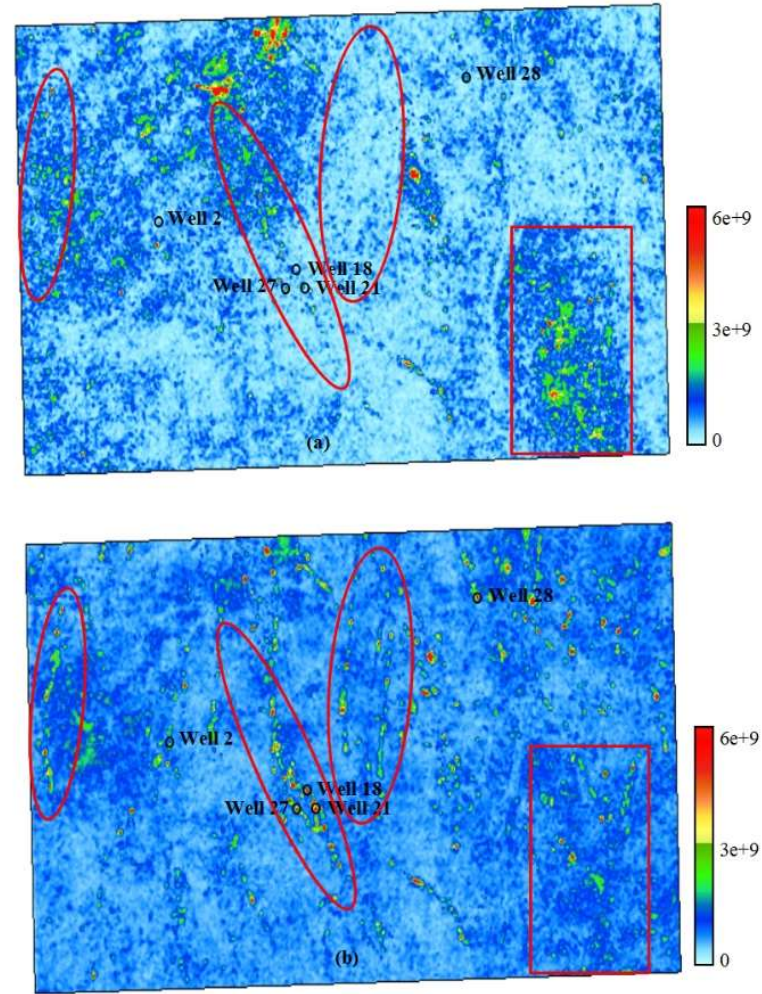

((a) spectral balancing; (b) without spectral balancing)

Fig. 5. Comparison of P-wave dispersion RMS attributes extracted 50ms down along Yijianfang group (To3t)

\section{Application}

\subsection{Fluid identification}

Figure 6 shows P-wave dispersion gradient of six wells and circles are for DST. It describes that P-wave dispersion gradient is comparatively stronger at Well 33 (gas) contained less water, whereas the strength at Well 35 (water) is weaker. Well 31 and Well 34 are both oil well,with similar porosity and saturation, manifesting similar degree of $\mathrm{P}$-wave dispersion gradient, which is slightly stronger than Well 32 (oil and water). Application effects bring into correspondence with theoretical theory of fluid dispersion response. Furthermore, the frequencydependent AVO inversion algorithm has a markedly higher degree of fluid recognition and significantly better application effect in igneous rock reservoirs.

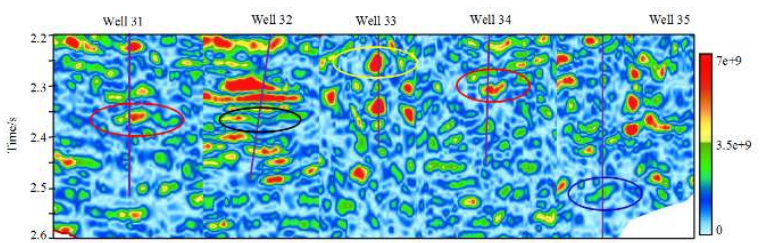

Fig. 6. Sections of inverted P-wave dispersion. 


\subsection{Lithology identification}

The lithology in the red box of figure 7 is explosive facies trachyte and trachyte breccia lava, which is the high-quality lithology sought in the study area. It is obvious that higher Pwave dispersion gradient value is displayed in well 36 and well 37, while well 38 and well 39 in figure 8 correspond to lower dispersion gradient value where the lithology is overflow facies basalt and explosive facies basalt, which is consistent with the display of root mean square attribute values on the plane (Fig. 9).

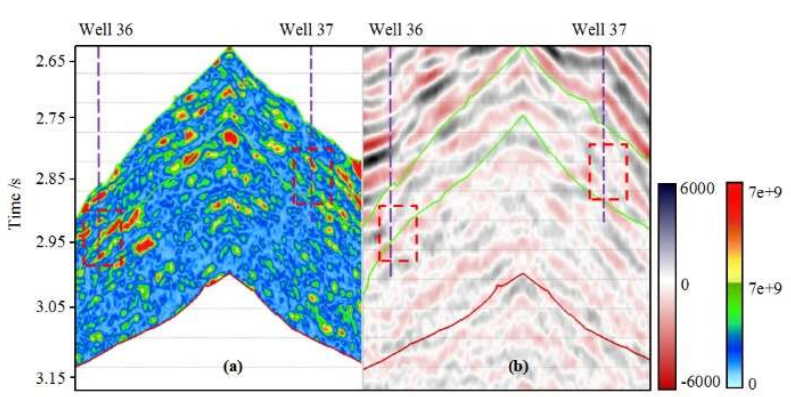

((a) P-wave dispersion gradient; (b) seismic amplitude)

Fig. 7. Comparison of sections

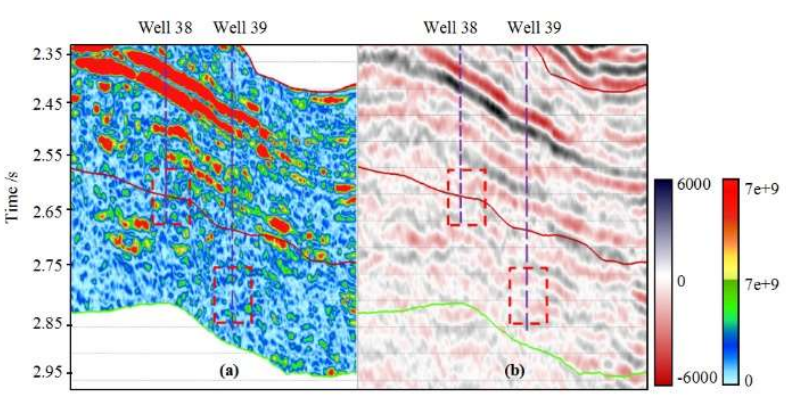

((a) P-wave dispersion gradient; (b) seismic amplitude)

Fig. 8. Comparison of sections

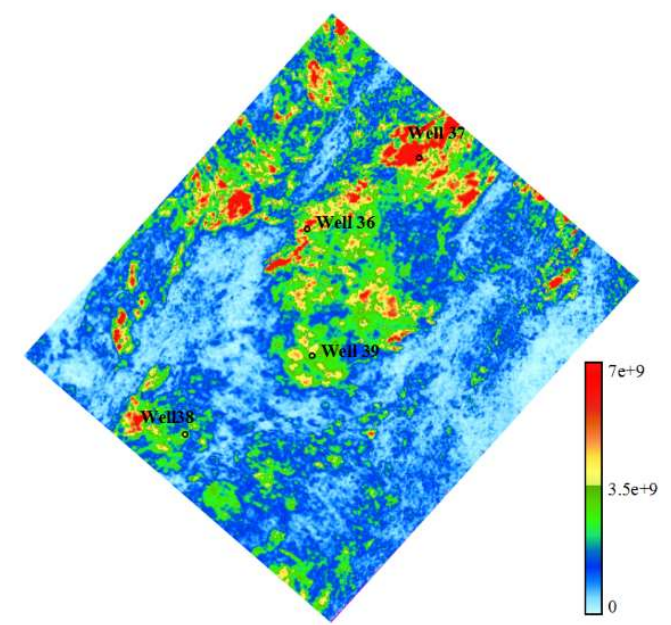

Fig. 9. Plane of P-wave dispersion RMS attribute

\section{Conclusion}

Based on the research of the frequency-dependent AVO inversion method, we primarily analyze the factors model and spectra balancing, hoping that the method can truly and effectively reflect the fluid type and identify highquality reservoirs. Then applied the improved method to igneous reservoirs. The result describes that the method can distinguish fluid types and the inverted P-wave velocity dispersion gradient can have a better recognition effect with high-quality reservoir lithology. In conclusion, the high recognition effect indicates that the frequencydependent AVO inversion method has high accuracy for fluid detection and high-quality reservoir identification, and improves the understanding of fluid detection and identification of complex igneous reservoirs.

\section{References}

1. W.P. Mason, H.J. McSkimin, Attenuation and scattering of high frequency sound waves in metals and glasses. The Journal of the Acoustical Society of America, 19, 3 (1947).

2. W.J. Toulis, Theory of a resonance method to measure the acoustic properties of sediments. Geophysics, 21, 2 (1956).

3. M. Chapman, Liu E., Li X.Y., The influence of fluidsensitive dispersion and attenuation on AVO analysis. Geophysical Journal International, 167, 1 (2006)

4. A. Wilson, M. Chapman, Li X.Y., Frequencydependent AVO inversion. SEG Annual Meeting, 79 (2009)

5. Wu X.Y., Reaserch on dispersion AVO inversion based on spectrum analysis technology. (Doctoral Dissertation, China University of Geosciences (Wuhan), Wuhan, China, 2010)

6. Sun S.Z., Yue H.Y., Hu L.G., Zhang Y.Y., Du. T., An Improved Frequency-dependent AVO Inversion Algorithm for Fluid Detection. SEG Annual Meeting, 84 (2014).

7. Du T., Sun S.Z., Zhang Y., Yue H.Y., Li C., Liu Z., A Study on Influence of Frequency selection to Frequency-dependent AVO Inversion. EAGE Conference and Exhibition, 76 (2014) .

8. Sun S.Z., Yue X.T., Liu L.F., Du T., Karst Carbonate Reservoir Identification Using Frequency-dependent AVO inversion in Tarim Basin. EAGE Conference and Exhibition, 78 (2016). 